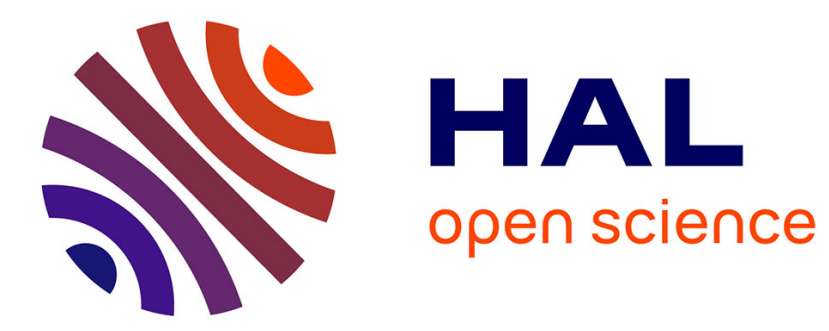

\title{
Les migrants transnationaux: une nouvelle figure sociale en Roumanie
}

Swanie Potot

\section{To cite this version:}

Swanie Potot. Les migrants transnationaux : une nouvelle figure sociale en Roumanie. Revue d'Etudes Comparatives Est-Ouest, 2002, 33 (1), pp.101-120. halshs-00080692

\section{HAL Id: halshs-00080692 \\ https://shs.hal.science/halshs-00080692}

Submitted on 20 Jun 2006

HAL is a multi-disciplinary open access archive for the deposit and dissemination of scientific research documents, whether they are published or not. The documents may come from teaching and research institutions in France or abroad, or from public or private research centers.
L'archive ouverte pluridisciplinaire HAL, est destinée au dépôt et à la diffusion de documents scientifiques de niveau recherche, publiés ou non, émanant des établissements d'enseignement et de recherche français ou étrangers, des laboratoires publics ou privés. 


\section{Les migrants transnationaux : une nouvelle figure sociale en Roumanie}

Swanie Potot ${ }^{*}$

Article paru dans la Revue d'Etudes Comparatives Est-Ouest, vol.33, $n^{\circ} 1$, mars 2002, pp.149178.

\section{Résumé :}

Cet article traite des migrations transnationales temporaires pratiquées par les classes moyennes roumaines.

Le texte décrit comment, dans le contexte de la «transition » post-socialiste qui semble ne pas aboutir, les migrations momentanées de travail vers des pays riches apparaissent, à côté d'autres tendances migratoires, notamment pendulaires ou liées au «commerce de ma valise », comme une stratégie particulière d'adaptation à la crise. S'appuyant sur différentes études statistiques, l'auteur constate que ce recours est de plus en plus usité par les jeunes citadins diplômés. En se basant sur une enquête menée auprès de ce public dans une ville moyenne proche de Bucarest, l'analyse se focalise ensuite sur la constitution des réseaux nécessaires à la mise en œuvre de ces mouvements informels à travers l'Europe. Elle montre alors qu'au-delà d'un projet initial ponctuel et exceptionnel, la migration tend à devenir, pour des individus possédant un capital social transnational de plus en plus vaste, une occupation à plein temps. La dernière partie de l'article examine la position sociale de ces migrants en Roumanie. Partageant leur existence entre une installation confortable sur place et une succession de «stages » de labeur à l'étranger, ils adoptent dans leur ville d'origine, un comportement et des pratiques spécifiques à leur condition ascendante, dessinant ainsi les contours d'une nouvelle catégorie sociale.

\footnotetext{
* Doctorante, allocataire de recherche au Soliis-Urmis, Université de Nice-Sophia Antipolis.
} 


\section{Article :}

La migration temporaire -et souvent clandestine- de travail vers les pays d'Europe de l'Ouest est un recours de plus en plus usité en Roumanie. La vulgarisation de cette pratique auprès des jeunes citadins diplômés mérite une attention particulière. Ce public n'est ni particulièrement indigent ni marginalisé dans son pays ; pourtant, ces jeunes gens acceptent de partir à l'étranger effectuer toutes sortes d'emplois dévalorisés. Durant ces séjours, ils économisent pour consommer ensuite le fruit de ce labeur en quelques mois dans leur ville d'origine.

Afin de saisir l'apparition de ce phénomène dans l'après 1989, il est utile, dans un premier temps, de se pencher sur le contexte socio-économique du pays. C'est en effet dans les stratégies de survie informelles développées par l'ensemble de la population, que l'on discerne les prémices des usages migrants. En Roumanie, les migrations transnationales ont revêtu, au cours de la dernière décennie, des formes multiples, portées par des publics disparates. Il faut donc également s'intéresser de près à l'évolution de ces mouvements pour situer les pratiques émanant aujourd'hui des classes moyennes urbaines.

Une série d'observations au sein d'une ville de départ permet de comprendre comment le phénomène migratoire prend forme dans son contexte local. La notion de capital social éclaire notamment les processus de sélection des candidats au départ autant que la « réussite » ou «l'échec» de la migration. Simultanément, l'étude du mode de vie des migrants fait apparaître une tendance de cette activité à se perpétuer. Née de projets ponctuels, elle se transforme rapidement en véritable carrière. Dans ce contexte, l'analyse montrera qu'être migrant revient, pour finir, à s'agréger à une nouvelle catégorie sociale en Roumanie.

La réflexion présentée s'appuie sur une recherche de trois années conduite auprès de la population migrante roumaine. Les différentes enquêtes qualitatives ont porté sur deux réseaux migratoires, ceux-ci étant compris, au sens de D.Massey, comme «l'ensemble des liens interpersonnels qui relient les migrants, les futurs migrants, et les non migrants dans les espaces d'origine et de destination, à travers les liens de parenté, d'amitié, et une origine communautaire partagée $»^{1}$. Le premier réseau étudié se déploie entre la province de Teleorman, dans le sud de la Roumanie, et le département d'Almeria en Espagne. Cette circulation a fait l'objet de deux enquêtes de terrain en Espagne, à El Ejido. La première,

\footnotetext{
${ }^{1}$ Massey (D.), "Theories of International Migration: Review and Appraisal" in Population and Development Review, 19(3), 1993 cité par Ma Mung et Al., La circulation migratoire. Bilan des travaux. Migrations études, $\mathrm{n}^{\circ} 84,1998, \mathrm{p} .15$.
} 
d'une durée de deux semaines au mois d'août 2000, a servi de phase exploratoire pour une seconde partie, au printemps 2001. En plus de quelques estimations chiffrées par les autorités espagnoles ${ }^{2}$, l'essentiel des données recueillies sont des entretiens semi-directifs de migrants Roumains et Marocains et des notes prises au cours d'entrevues avec responsables municipaux ou d'associations. Cette migration a également fait l'objet d'une troisième enquête dans la région d'origine, sur laquelle je reviendrai.

L'autre volet de cette étude, qui constitue la matière principale du présent article, porte sur un parcours migratoire constitué à partir d'une ville moyenne de Roumanie, Târgoviste, dont la destination principale fut, dans un premier temps (de 1994 à 1999) la Côte d'Azur française où, d'après mes estimations, environ 150 migrants originaires de cette ville se sont succédés. A partir de 1999, cette destination a été petit à petit délaissée, et ils se sont repliés sur Londres où il y aurait, à l'automne 2001, plusieurs centaines de migrants de Târgoviste. Ce réseau a fait l'objet d'une étude approfondie sur la base d'entretiens, de récits de vie mais aussi d'observations participantes sur les différents lieux qui constituent cet espace migratoire. A Nice, j'ai mené une enquête intermittente d'environ une année, en 1998, auprès des acteurs du réseau mais aussi de leurs employeurs et de leurs logeurs. Cela a donné lieu à une trentaine d'entretiens enregistrés et à de nombreuses observations portant sur la vie quotidienne et les pratiques de ces acteurs. A Londres, un séjour de quatre semaines parmi les migrants en octobre-novembre 2001 a permis d'y effectuer des entretiens, et d'avoir de multiples conversations qui ont systématiquement fait l'objet de notes. En Roumanie, deux enquêtes dans la ville d'origine, l'une de 15 jours en 1999, l'autre de trois mois au printemps 2000, ont non seulement approfondi la connaissance de ce réseau migrant, mais m'ont également permis d'obtenir de nombreux renseignements sur les pratiques informelles plus généralement liées aux migrations en Roumanie. Au sein du réseau étudié, j'ai réalisé environ vingt interviews auprès de migrants présents en Roumanie à ce moment-là et quelques-uns auprès de membres de famille de personnes alors à l'étranger. Ces entretiens, bien qu'instructifs, ne rendaient que partiellement compte des activités et des pratiques de cette population. C'est pourquoi ils furent complétés par des observations directes. Je connaissais, pour les avoir rencontrés en France, une quinzaine de migrants sur place lors de mon arrivée et j'étais logée dans une famille dont deux des trois enfants se trouvaient à l'étranger. Cela m'a permis de fréquenter quotidiennement le groupe des migrants et de leurs proches auquel je fais référence dans la deuxième partie du texte. Les soirées passées à leurs côtés aux

\footnotetext{
${ }^{2}$ Par définition, il est très difficile d'obtenir des statistiques fiables sur les migrations clandestines.
} 
terrasses de cafés, les conversations informelles que j'ai pu avoir au cours de sorties ou de repas communs ou mêmes certaines interactions entre des migrants dans lesquelles je n'étais pas impliquée mais dont j'ai été témoin, ont alors fait l'objet d'une prise de notes minutieuse. Le groupe d'étude concernait une centaine de personnes pratiquant, pour la majorité, de continuelles allées-venues entre l'Union Européenne et la Roumanie. J'ai rencontré environ la moitié de cette population et j'ai eu des contacts répétés avec un cercle d'une vingtaine de personnes. C'est de l'ensemble de ces données qu'est issue l'analyse présentée dans les lignes qui suivent.

\section{La Roumanie, une économie en transition ?}

Décembre 1989 marque pour le peuple roumain la fin d'un des régimes les plus autoritaires du système communiste. Mais l'on se souvient également aujourd'hui de la « révolution» comme de l'événement qui a marqué le début de la crise socio-économique engendrée par la transition. Dans les premières années qui ont suivi ces évènements, la récession a semblé naturelle, l'adoption de l'économie de marché avait un coût social qui, une fois dépassé, devait être compensé par la croissance et le développement de la société de consommation. Mais, ni les aides des institutions internationales, tels le Fonds Monétaire International ou la Banque Mondiale, corrélées à des programmes de réformes économiques, ni l'alternance politique n'ont conduit à cette deuxième phase. Après douze années, le passage à une économie capitaliste viable ne s'est toujours pas opéré. Même si les indicateurs économiques semblent plus optimistes en 2000 et 2001 grâce à une reprise de la croissance ${ }^{3}$, les conditions de vie actuelles sont aujourd'hui inférieures à celles de 1989. Les rêves d'occidentalisation rapide du pays ont petit à petit laissé la place à des préoccupations matérielles de premier ordre. Les difficultés rencontrées au jour le jour poussent même une partie des Roumains à considérer aujourd'hui que, malgré la dictature, «c'était mieux avant $»^{4}$. Le taux d'abstention élevé ainsi que le score du parti extrémiste aux élections de novembre-décembre $2000^{5}$ souligne, s'il en était besoin, le désenchantement de la population.

\footnotetext{
${ }^{3}$ En 2000 la croissance était de 1,6\% et atteignait les 4.5\% en 2001. Ici comme dans la suite du texte, sauf indication contraire, les chiffres cités proviennent de l'Institut National des Statistiques Roumaines, www.insse.ro.

${ }^{4}$ Le niveau de vie en 2000 représenterait 80\% de celui de 1989, cf. Lhomel (E.), « Roumanie 2000-2001 Un nouveau départ? $»$, note 8, p. 173 .

${ }^{5}$ Lors des élections législatives et présidentielles du 26 novembre le taux d'abstention était supérieur à $43 \%$ et le Parti de la Grande Roumanie, d'extrême droite, s'imposait comme deuxième formation politique du pays avec
} 
Les efforts de celle-ci s'orientent alors vers la satisfaction des besoins privés. Avec une inflation des produits alimentaires de plus de $40 \%$ et un salaire moyen de 108 Euros en 2000, nombreux sont les ménages qui ne parviennent plus à assurer leur minimum vital. L'objectif principal est de maintenir le niveau de vie de sa famille.

Cette situation a eu pour conséquence de développer plus encore une économie informelle déjà florissante sous le communisme. En effet, comme le remarque D.Kideckel ${ }^{6}$, déjà à l'époque de Ceausescu les échanges non contrôlés étaient monnaie courante. Ils permettaient de palier les insuffisances de l'économie planifiée et de maintenir un certain standard de vie. C'est dans le prolongement de ces pratiques anciennes qu'il faut comprendre l'ampleur de l'économie parallèle actuelle. Sous le communisme, celle-ci se concentrait majoritairement sur les échanges non marchands et le trafic de produits de consommation courante. Avec l'apparition du capitalisme, ces échanges se sont dynamisés et étendus à de nouveaux secteurs. Une étude datant de 1996 estimait que le secteur informel représentait plus de la moitié de la consommation des ménages (46511 milliards de lei pour un total de 77733 milliards $)^{7}$.

Le taux de chômage, qui a rarement dépassé les $11 \%$ depuis 1989 , ne peut expliquer à lui seul cet essor. Si, sous la dictature, l'économie informelle était imputée à une situation de manque de produits sur le marché légal, ce sont aujourd'hui les défaillances organisationnelles du système officiel qui sont mises en cause. Le recours à la deuxième économie est encouragé par le désordre administratif et la variabilité des cadres juridiques qui ont suivi le changement de régime ${ }^{8}$. Etant donné la complexité des procédures administratives et le degré de corruption des services publics, il est devenu plus simple et moins coûteux pour les particuliers de mener leurs échanges sur le marché noir. Cette alternative permet de contourner les contraintes structurelles d'une administration corrompue et désorganisée. Par ailleurs, le salaire moyen, qui avoisine les 160 Euros (septembre 2001), ne permet pas de vivre décemment. D'après la plaquette d'expatriation publiée par le ministère des Affaires Etrangères français, le coût moyen de la vie était d'environ 3000FF (soit 457 Euros) par mois en 1998. Les écarts sont alors compensés par l'économie parallèle. Ainsi, le marché informel

$20,98 \%$ des sièges au parlement et $33,17 \%$ des voix pour son candidat au deuxième tour de l'élection présidentielle.

${ }^{6}$ Kideckel (D.), The Solitude Of Collectivism, p.69.

${ }^{7}$ Duchêne (G.), « Les revenus informels en Roumanie: Estimation par enquête », p.40.

${ }^{8}$ Idem, p.36. 
n'est pas l'apanage des mafieux ou des exclus du système; il s'agit d'un usage courant, à l'œuvre dans l'ensemble de la société.

L'économie parallèle couvre de nombreux domaines qu'il convient d'expliciter afin de comprendre son lien avec les migrations transnationales. G.Duchêne propose une définition globale des activités informelles: celles-ci «peuvent être définies comme l'ensemble des activités économiques légales ou illégales entreprises par des unités de petite taille (individuelle ou familiale) donnant lieu à une production au sens du Système des Comptes Nationaux et qui sont opérés en marge des systèmes socio-fiscaux et réglementaires imposés par l'Etat $»^{9}$. Sans prétendre être exhaustif, on peut dresser une typologie des activités les plus usitées en Roumanie, en fonction du degré d'implication qu'elles requièrent. Une part importante de cette économie ne fait pas directement intervenir la notion d'argent. Il s'agit en premier lieu de l'auto-consommation. Comme à l'époque communiste, de très nombreux ménages pratiquent une petite activité agricole à usage privé qui s'ajoute en général à un emploi salarié. Cette production implique également des trocs de produits de même nature avec l'entourage plus ou moins proche. Sur le même mode, dans des cercles relativement restreints, on peut échanger des services ou de menus travaux dont le paiement repose sur une réciprocité dans le temps. Ces différentes formes d'entraide ne constituent pas, proprement dit, des revenus secondaires ; elles apparaissent en complément d'activités lucratives.

Dans le même temps, certains pans de l'économie marchande se développent en marge des institutions. Le secteur agricole est spécifique dans la mesure où les ventes sur les marchés paysans ne sont que rarement déclarées ${ }^{10}$. Ce secteur appartient donc, de fait, à l'économie informelle. En revanche, beaucoup d'entreprises individuelles sont plus ambiguës. Garagiste, tailleur, peintre ou enseignant, sont autant de professions qui souvent s'exercent partiellement ou totalement en dehors de tout contrôle étatique. Il s'agit alors véritablement de travail au noir qui peut intervenir soit en tant qu'activité principale (première source de revenu) soit en complément d'un emploi salarié ou bien du chômage. Le recours à une deuxième activité de ce type n'appartient pas à des catégories spécifiques, il se rencontre dans un large éventail de la population active.

Enfin, le commerce apparaît comme une composante importante de la seconde économie. C'est en général à ce secteur que l'on fait référence lorsque l'on parle de « trafics ». Malgré ce terme, l'essentiel de ce marché concerne des produits dont la vente n'est

\footnotetext{
${ }^{9}$ Idem, p.35.

${ }^{10}$ Idem, p.40.
} 
pas illicite. Ce sont les conditions de commercialisation qui transgressent les lois. Il peut s'agir, notamment, de marchandises sorties illégalement de grandes entreprises pour être revendues par des particuliers. Sous le communisme, ces trafics liés aux vols dans les firmes d'Etat étaient fréquents; ils s'opèrent aujourd'hui sur le même mode, bien que dans des proportions sans doute plus faibles. Un deuxième procédé consiste à acheter légalement des stocks de marchandises à des fabricants puis à les débiter sur les marchés ou à d'autres intermédiaires en évitant les taxes et contrôles de l'Etat.

Un autre type de négoce, directement lié à la circulation individuelle, a pris une nouvelle impulsion avec l'ouverture des frontières. Il s'agit du «commerce de la valise ». Tandis que ces petits trafics d'import-export avec les pays limitrophes étaient extrêmement risqués à l'époque de la dictature, ils se sont vulgarisés après 1990. C'est notamment avec la Turquie, la Hongrie et la Serbie qu'ils sont actuellement le plus développés. Ces petites entreprises sont soit le fait d'individus agissant seuls; soit quelques personnes, rarement plus de deux ou trois, collaborent pour se répartir les tâches. Le principe est de vendre en Roumanie des produits que l'on est soi-même allé chercher sur les marchés étrangers. Les cassettes audio ou les vêtements en provenance d'Istanbul en sont un exemple bien connu ${ }^{11}$. Les allées-venues se font généralement en bus ou en train et les séjours à l'étranger ne dépassent pas plus d'un jour ou deux. Situées au carrefour des migrations transnationales et du commerce local, ces affaires mettent à profit l'espace international pour résister à la conjoncture défavorable.

\section{Les migrations transnationales comme alternative}

C'est dans cet esprit qu'il faut comprendre les migrations transnationales abordées dans la suite du texte. Mais clarifions d'abord rapidement ce que l'on entend par «migrations transnationales ». Selon B.Badie, « s'interroger sur les réseaux transnationaux (...) consiste à analyser la construction des liens sociaux qui donnent réalité et vie à la mondialisation ${ }^{12}$. Sans s'aligner sur l'idée A.Portes ${ }^{13}$ selon lequel les migrations apparaissent en marge, ou en réponse, à la globalisation de l'économie, on considère ici qu'elles appartiennent à un même processus d'échanges et de sociabilités qui, de plus en plus, s'affranchit des limites tracées par les frontières nationales. Comme le souligne encore B.Badie, le pouvoir des Etats sur la scène

\footnotetext{
${ }^{11}$ Voir à ce sujet l'article de Aktar C. et Ögelman N., « Recent Developments in East-West Migration : Turkey and the Petty Traders ».

${ }^{12}$ Badie (B.), « Préface » in Sociologie des réseaux transnationaux, p.15.

${ }^{13}$ Portes, « La mondialisation par le bas ».
} 
internationale tend à perdre de son hégémonie pour laisser une place grandissante à des stratégies d'acteurs autonomes. Qu'il s'agisse des multinationales, ou pour reprendre les termes d'A.Portes au sujet des communautés transnationales de migrants, de «la mondialisation par le bas », des réseaux sociaux se déploient de plus en plus dans des espaces multipolaires. Cela ne revient nullement à postuler que, pour les migrants, les frontières, où les disparités spatiales sociales et économiques n'ont plus d'effet. Au contraire, comme nous allons le voir, la migration transnationale repose sur une capacité spécifique de certains acteurs à mettre en liaison des localités dissemblables et à passer de l'une à l'autre. Les réseaux migrants opèrent ainsi des «constructions territoriales » ${ }^{14}$ liant différents lieux de passage qui, comme l'écrit A.Tarrius, ne se situent «ni ici ni là, mais ici et là en même temps $»^{15}$. C'est bien parce qu'ils sont inscrits simultanément dans plusieurs localités, par ailleurs dissociées, que la position des migrants est avantageuse. L'intérêt de celle-ci passe justement par le décalage existant entre ces sociétés. Comme le souligne Piore ${ }^{16}$, l'avantage saisi pas les migrants ne tient pas seulement au différentiel de richesse qui, dans le cas présenté, permet d'obtenir à l'Ouest de faibles salaires qui seront dépensés en Roumanie, démultipliant ainsi leur valeur, mais également à une distance sociale. C'est, parallèlement, parce que le statut social dévalorisé qui leur est imposé à l'étranger n'a pas d'incidence sur ce qui est vécu dans la société d'origine que le sacrifice est possible. Mais la référence au transnationalisme ne renvoie pas seulement à une dimension géographique dans laquelle les acteurs sont plus autonomes. Elle appelle également, comme le suggère la deuxième partie de cet article, à s'intéresser aux espaces de sociabilité qui sont les supports de ces mouvements. Ceux-ci sont caractérisés par «une horizontalité des rapports sociaux [qui], peu institutionnalisés (...), se constituent au sein de sphères informelles $»^{17}$. Sans éliminer les dépendances liées au pouvoir, il n'y existe pas, en effet, de hiérarchie pré-établie. Les relations, moins codifiées que dans les structures institutionnelles, sont toujours susceptibles d'évoluer, inversant parfois dans le temps les rapports de domination. Au sein de ces réseaux, comme dans les échanges qui les lient aux sociétés qu'ils traversent, les négociations et les relations interpersonnelles ont une importance première, avant toute forme de réglementation rigide. Ainsi, parler de migrations roumaines transnationales renvoie tant aux pratiques de

\footnotetext{
${ }^{14}$ Tarrius (A.), « Territoires circulatoires et espaces urbains », p.51.

${ }^{15}$ Ibidem.

${ }^{16}$ Piore (M.J.), "The Shifting Grounds for Immigration".

${ }^{17}$ Colonomos (A.), « La sociologie des réseaux transnationaux », in Sociologie des réseaux transnationaux, p.22.
} 
mobilité circulatoire, faites de continuels mouvements entre la ville d'origine et plusieurs pôles migratoires dans l'espace européen, qu'aux formes d'organisation et de structuration des relations sociales sur lesquelles reposent cette circulation.

En Roumanie, ces migrations doivent, à mon sens, être comprises comme le prolongement des stratégies individuelles développées par les Roumains dans le but de résister au déclin de leur condition. Comme l'écrit M.Morokvasic, «il s'agit de gens qui réagissent à une situation de crise en se mettant en route, pour éviter de devenir les laisséspour-compte de sociétés engagées dans un processus de transformation rapide et, surtout, imprévisible $»^{18}$. Aller chercher ailleurs les moyens de mieux vivre ici est le principe qui guide ces pratiques. Pour D.Sandu, les déplacements temporaires de travail vers des pays plus riches sont à mettre en relation avec une habitude plus ancienne de «navettes » villecampagne ${ }^{19}$. Le «navettisme $»^{20}$ permettait aux habitants des zones rurales d'améliorer leurs conditions de vie en allant travailler en ville, sans entrer de plain-pied dans le circuit économique urbain qui, étant éloigné des centres de production alimentaire, était, sous la dictature, plus dépendant que celui des campagnes. Le parallèle avec la mobilité internationale semble tout à fait probant. Il s'agit en effet d'étendre ses activités à un nouveau territoire, sans se couper économiquement et socialement de l'ancien.

En ce sens, la circulation transnationale n'est pas un phénomène qui romprait l'équilibre local. On doit plutôt y voir une forme particulière d'adaptation à la crise. C'est de cette façon qu'elle est vécue par la population roumaine qui envisage la migration temporaire plus comme une alternative aux «débrouilles » à l'œuvre sur place que comme un moyen d'échapper définitivement à la condition roumaine. C'est parce qu'elle s'inscrit dans une continuité que l'idée de «Faire une saison ${ }^{21}$ à l'Ouest est devenue si populaire. Selon le

\footnotetext{
${ }^{18}$ Morokvasic (M.), «Entre l'Est et l'Ouest, des migrations pendulaires » in Migrants. Les nouvelles mobilités en Europe, p.123.

${ }^{19}$ Sandu (D.), « Les enjeux des réseaux migratoires dans l'espace social de la transition ».

${ }^{20}$ Le néologisme renvoie au concept roumain de navetism.

21 Cette expression, qui relate bien l'état d'esprit guidant ces migrations temporaires, a été choisi par D.Diminescu et R.Lagrave comme titre d'un travail sur la circulation de migrants entre le pays d'Oas (Roumanie) et Paris : Diminescu (D.) et Lagrave (R.), « Faire une saison. Pour une anthropologie des migrations roumaines ».
} 
Baromètre d'Opinion Publique ${ }^{22}$, en $2000,18 \%$ de la population roumaine prévoyait d'aller travailler temporairement à l'étranger. Le fait de migrer est, pour les Roumains, un recours qui demande un peu plus d'investissement que les « trafics » habituels mais dont, en échange, on escompte des retombées plus importantes. De même, les restrictions légales auxquelles les passages de frontières sont soumis apparaissent comme des aléas à surmonter plus que comme de véritables barrières à la migration. La Roumanie, jusqu'au $1^{\text {er }}$ janvier 2002, était en effet soumise à un régime de circulation particulier en Europe. Jusqu'alors l'Union Européenne, à travers l'Espace Schengen, gardait ses portes fermées aux ressortissants roumains, imposant, pour les seuls voyages touristiques autorisés, l'obtention de visas délivrés au compte-goutte ${ }^{23}$. Même avec l'autorisation de circuler apparue récemment, les migrants n'ont pas le droit de travailler à l'étranger, si bien que les migrations de travail à l'Ouest relèvent souvent de pratiques illégales.

Ce que l'on désigne ici comme migration est donc à distinguer de l'émigration, plus marginale, autant que des déplacements dus au «commerce de la valise ». Il s'agit de séjours de quelques mois à une ou deux années durant lesquels le migrant exerce une activité lucrative à l'étranger, généralement en Europe de l'Ouest, parfois en Israël. Ces migrations sont caractérisées par une circulation accrue et par leur capacité à suivre les fluctuations du marché international du travail réservé aux migrants -avec ou sans papiers. Comme nous le verrons, une destination favorisée aujourd'hui peut être abandonnée demain au profit d'un nouveau secteur d'embauche dans un autre pays. Le seul point fixe dans le parcours migratoire reste le pays de départ qui devient alors également le pays des retours. En ce sens, il est plus pertinent de catégoriser ces migrations transnationales par les réseaux qu'elles mobilisent, liés à des territoires plus ou moins circonscrits en Roumanie, que par les lieux fluctuants dans lesquels elles se déploient à l'étranger.

\footnotetext{
${ }^{22}$ Fundatia pentru o societate deschisa, Barometrul de opinie publica, p.44. Sondage annuel effectué par téléphone sur 1816 personnes représentatives de la population roumaine. Détails et base sont consultables sur le site www.osf.ro

${ }^{23}$ Cette situation a pris fin le $1^{\mathrm{er}}$ janvier 2002, date depuis laquelle les ressortissants roumains peuvent effectuer des séjours de courte durée (ne dépassant pas 90 jours) dans l'Espace Schengen sans visa. Cependant, en plus de leur passeport, ils doivent faire la preuve, lors de leur sortie de la Roumanie et de leur entrée dans l'Espace Schengen qu'ils possèdent une assurance médicale, un billet de transport aller-retour et un montant de 100 euros par jour de voyage.
} 


\subsection{Des pratiques migratoires diversifiées}

D'une façon globale, et pour situer l'étude de cas qui suit, on peut dresser un rapide panorama chronologique de ces migrations depuis $1989^{24}$.

Dans un premier temps, les mouvements se sont surtout intensifiés vers les pays limitrophes. Les séjours saisonniers de travail en Hongrie, Yougoslavie et Turquie (qui n'est pas un pays limitrophe mais avec lequel les échanges sont nombreux) se sont multipliés au début de la décennie. Ils concernaient surtout les populations frontalières qui avaient déjà établi des liens avec ces régions, et sont apparus en marge des voyages commerciaux.

C'est à partir des années 1993-94 que les départs vers des pays plus lointains et plus difficiles d'accès ont commencé à se populariser. Deux types de migrations se sont juxtaposés. D’une part, plusieurs pays, notamment l'Allemagne et Israël ont recruté de la main d'œuvre en Roumanie. Dans le cadre d'accords de «Werkertrage», des firmes allemandes ont passé des contrats avec des firmes roumaines qui fournissaient non seulement des travailleurs mais également des matériaux nécessaires à certains chantiers de construction. Ces échanges ont surtout fonctionné entre 1993 et 1996 mais ont quasiment disparu aujourd'hui. De même les autorités Israéliennes décident, chaque année, du quotas de « travailleurs invités » (guest workers) par nationalité. Les Roumains, qui représentaient 29\% des travailleurs étrangers en situation légale en 2000, y étaient le groupe le plus représenté ${ }^{25}$. Ces recrutements, effectués depuis la Roumanie, passent toujours par des agences roumaines qui servent d'intermédiaire entre les candidats au départ et les employeurs privés étrangers. Le voyage est alors financé soit par l'agence de recrutement soit par l'entreprise elle-même. De plus, cette dernière s'engage, en général, à loger les travailleurs sur place. Concrètement, cela s'est traduit pour les Roumains par la possibilité d'obtenir, depuis la Roumanie, un contrat temporaire de migration dans lequel tout était compris, le migrant étant totalement pris en charge. L'avantage est évident pour des individus qui n'avaient aucune connaissance de l'étranger. De ce fait, l'intérêt porté à ces recrutements a donné lieu à de nombreux trafics. Bien qu'elles soient rétribuées par les sociétés étrangères, les agences roumaines en charge de l'enrôlement des travailleurs ont systématiquement vendu ces contrats à ces derniers. Le

\footnotetext{
${ }^{24}$ Cette partie s'appuie sur les interventions de V.Rey, D.Sandu, D.Diminescu, B.Michalon, Z.Rozenhek, J.Arango, S.Potot, S.Gangloof et S.Weber lors du séminaire «Les circulations migratoires roumaines après 1989 » à la Maison des Sciences de l’Homme de Paris, les 23-24 novembre 2001.

${ }^{25}$ Ces statistiques, parues dans The Internet Jerusalem Post du 30 octobre 2001, ne tiennent pas compte des travailleurs palestiniens dont le statut est particulier.
} 
nombre en étant limité ${ }^{26}$, les agences ont pu faire monter les prix du recrutement sur le marché noir. Ainsi, certaines places pouvaient se vendre jusqu'à 2500 dollars, tandis que le salaire mensuel garanti par ces contrats avoisinait seulement les 1000 dollars. L'attrait que présentaient ces recrutements auprès du public s'explique de plusieurs façons. En premier lieu, au moment où ce type d'opportunité est apparu, les migrations non-pendulaires (c'est-àdire ayant une durée plus longue et s'inscrivant dans un espace plus large) en étaient à leurs balbutiements. Les réseaux migratoires, qui par la suite faciliteront la circulation, n'étaient pas encore en place et la migration apparaissait encore comme une aventure relativement risquée (dans la mesure où elle pouvait coûter plus que ce qu'elle rapporterait). Les contrats offraient alors la possibilité de partir avec l'assurance de pouvoir rentabiliser son voyage et d'être hébergé.

Par ailleurs, dès 1995, ces migrations temporaires sous contrat ont commencé à servir des initiatives plus autonomes. Cela apparaît encore aujourd'hui d'une façon plus marginale. Certains travailleurs sous contrat à l'étranger maximisent leurs revenus en cumulant les emplois. En plus du travail pour lequel ils ont été embauchés, ils s'adonnent à de petits travaux au noir durant leurs jours de repos ou après leur journée de labeur. Ainsi, la valeur de ces contrats auprès du public roumain dépend notamment du contrôle exercé sur les ouvriers en dehors de leurs heures de travail. Par exemple, un logement en foyer sur le lieu même du chantier n'est pas apprécié car il ne laisse pas au travailleur la possibilité de s'affranchir de la tutelle de son employeur. Ces recrutements peuvent également servir de laissez-passer pour des migrants entièrement autonomes. Après avoir fait le voyage avec la compagnie et parfois travaillé quelques temps auprès de l'employeur officiel, certains migrants quittent ce dernier pour trouver des propositions plus intéressantes sur place. Les salaires des «travailleurs importés » sont en effet toujours très faibles; les prélèvements censés compenser les frais de logement et/ou de nourriture réduisent encore ceux-ci. Une fois entré dans le pays, les migrants peuvent aisément trouver des conditions plus avantageuses sur le marché du travail national. Le contrat initial présente alors deux avantages notables : d'abord, le migrant peut prendre le temps de découvrir son lieu de travail et d'analyser les possibilités d'emploi extérieur qu'il offre, tout en ayant une activité assurée. Quelques fois, il garde sa place sur le chantier plusieurs mois avant de se lancer ailleurs. D'autre part, le recrutement lui attribue un

\footnotetext{
${ }^{26}$ Israël, qui est l'importateur de main d'œuvre roumaine le plus important, a fait entrer environ 40000 travailleurs roumains au cours des années quatre-vingt-dix. Voir à ce sujet l'article de Bartram (D.), "Foreign workers in Israel: History and Theory".
} 
droit de séjour qui lui permet de rester légalement dans le pays jusqu'à la fin de son contrat. Enfin, comme le note le Central Bureau of Statistics israélien, il arrive fréquemment qu'à la fin de ces contrats, les travailleurs ne rentrent pas immédiatement dans leur pays d'origine ${ }^{27}$. Après plusieurs mois passés sur place en toute légalité, ils ont une bonne connaissance des lieux et peuvent envisager de rester encore quelques temps clandestinement dans le pays.

Cela explique partiellement que les migrations autonomes, le plus souvent clandestines, se soient, dans un premier temps, développées dans les mêmes espaces que les migrations organisées légalement. C'est au départ sur leurs traces que sont apparus des migrants qui n'étaient liés à aucun contrat. La destination privilégiée des mouvements clandestins fut au départ, dès 1993-94, l'Allemagne. Les migrants contractuels ont parfois servi de relais pour des compatriotes autonomes. Même sans apporter véritablement une aide sur place, ils furent le support de liens entre leur région d'origine et leur destination d'accueil. Diffusant des informations à leurs familles et à leurs proches sur leur expérience à l'étranger, ils favorisaient implicitement les voyages dans ces régions. De la même façon, les Aussiedler ${ }^{28}$ récemment émigrés de Roumanie ont jeté des ponts entre certaines régions d'Allemagne et la Transylvanie d'où ils étaient originaires ${ }^{29}$, de telle sorte que les premiers migrants indépendants ont suivi les mêmes parcours. En ce sens, les migrations légalement encadrées ont orienté les pratiques de circulations clandestines à leur début.

Par la suite, ces migrations se sont développées dans d'autres pays européens. Cette ouverture était facilitée par le concept même de l'Espace Schengen. La convention qui lie les pays signataires ${ }^{30}$ prévoit en effet de supprimer les contrôles sur les personnes, quelle que soit leur nationalité, aux frontières intérieures en harmonisant les contrôles aux frontières extérieures et en élaborant une politique commune en matière de visa. De ce fait, après le

\footnotetext{
${ }^{27}$ CBS, mentionné dans The Internet Jerusalem Post du 30 octobre 2001.

${ }^{28}$ Le concept d'Aussiedler, tel qu'il fut défini par la «Loi sur les réfugiés et les personnes déplacées » de 1953 (Bundesvertriebenen -und Flüchtlingsgesetz), désigne, en Allemagne, les immigrants issus de minorités allemandes d'Europe centrale, orientale et de l'ex-URSS, dont on reconnaît, à ce titre, l'appartenance à la nation allemande.

${ }^{29}$ Sur ce point, voir l'article de Michalon (B.), « De la politique des Aussiedler à la circulation : diversification des pratiques migratoires des Saxons de Transylvanie».

${ }^{30}$ Les pays signataires de la Convention de Schengen, constituant «l'Espace Schengen », sont : l'Allemagne, l'Autriche, la Belgique, le Danemark, l'Espagne, la Finlande, la France, la Grèce, l'Italie, le Luxembourg, les Pays-Bas, le Portugal, la Suède et, bien que ces deux pays n'appartiennent pas à l'Union Européenne, la Norvège et l'Islande.
} 
passage de la première frontière, les migrants n'ont plus de difficulté pour circuler d'un Etat à l'autre. Ainsi, différents pays ont pu être explorés de façon à découvrir les destinations particulièrement attractives. De ce fait, lorsque l'Allemagne, à partir de 1997, a augmenté ses contrôles et restreint ainsi le travail au noir, ou lorsque la France, en 1998, limitant les demandes d'asile ${ }^{31}$, a réduit les possibilités de rester légalement sur son sol, les migrants ont délaissé ces pays sans pour autant mettre fin aux migrations en Europe. A partir de 1995, l'Italie a également attiré beaucoup de travailleurs roumains de passage; par la suite l'Espagne ou depuis peu le Royaume-Uni (hors Espace Schengen) sont devenus des destinations particulièrement prisées.

L'élection d'un site comme pôle migratoire dépend d'une pluralité de facteurs. Le plus important est sans aucun doute la rentabilité de la migration; autrement dit, il est indispensable pour les migrants d'avoir la possibilité de travailler sur place. En cela, leur arrivée doit coïncider avec leur exploitation potentielle sur le marché du travail du pays dit d'accueil. Dans le Sud de l'Espagne notamment, si les migrants roumains sont nombreux, c'est parce que l'agriculture locale crée un facteur pull important. Depuis quinze ans, l'immigration clandestine dans la région est largement exploitée par ce secteur qui, imposant de faibles salaires à ces travailleurs sans droits, parvient à réduire ses coûts de production et ainsi à se placer en tête de la vente de produits frais en Europe. Hormis le travail domestique qui emploie un certain nombre de femmes, les Roumains illégaux n'ont pas d'autre domaine d'embauche dans la province d'Almeria. En ce sens, il apparaît clairement que leur venue dans cette région est corrélée aux opportunités offertes par le secteur agricole. De même, les migrants rencontrés à Londres travaillaient principalement dans le bâtiment et l'hôtellerierestauration. Les emplois qu'ils occupent sont la plupart du temps attribués à des étrangers. La répression du travail clandestin étant peu développée au Royaume-Uni, ces migrants n’ont pas de difficultés à se faire employer aux côtés des personnes en situation régulière. Cependant, c'est parce que ces secteurs sont ouverts aux populations étrangères que les Roumains y trouvent une place. Le cas observé sur la Côte d'Azur illustre bien l'entente implicite qui préside à la constitution d'un parcours migrant: durant quatre années, les migrants de Târgoviste pratiquaient la vente d'un journal de rue ${ }^{32}$ implanté à Nice. Cette activité leur

\footnotetext{
${ }^{31}$ Suite à la loi dite « Loi Chevènement » du 11 juin 1998, les demandes d'asiles émanant de citoyens roumains sont traitées en procédure d'urgence, soit en moins de 10 jours, et assorties d'un rejet quasi-systématique.

${ }^{32}$ Il s'agit de journaux créés dans le but d'aider à la réinsertion sociale des plus démunis, vendus à la criée par des personnes en situation précaire dans les lieux publics.
} 
permettait non seulement de subvenir à leurs besoins sur place, mais également de constituer de petites économies en vue de leur retour. Le travail au noir sur des chantiers complétait parfois ces revenus mais les places étant peu nombreuses, les Roumains ne parvenaient pas à s'y faire employer à plein temps. C'est donc la première activité qui assurait la réussite de la migration. A partir de 1997, le succès des journaux de rue auprès du public français a commencé à décliner. En 1998, afin de redorer son image de marque, la publication présente à Nice a mis fin à l'embauche de clandestins parmi ses vendeurs. Dès lors, le public étudié a perdu son occupation principale. La conséquence au niveau du réseau ne s'est pas faite attendre : celui-ci a abandonné la destination niçoise au profit des régions de Milan et de Londres, qui offraient d'autres opportunités.

Le deuxième point essentiel tient à la connaissance collective du lieu. Seuls les migrants les plus avertis se permettent de conquérir de nouveaux territoires; la majorité se rend sur des positions déjà apprivoisées par le réseau auquel ils sont connectés. Ils y ont déjà des amis ou bien ont glané auprès de proches les informations utiles concernant une destination particulière. C'est alors la mémoire collective du groupe auquel le migrant est lié qui est mise à profit pour orienter les pratiques de celui-ci. L'expérience de chacun vient alors enrichir ce stock de connaissances partagé, ce qui favorisera l'orientation des nouveaux dans la même direction. En conséquence, les migrants se répartissent par niches ${ }^{33}$ dans l'espace européen, investissant certains territoires et en délaissant d'autres.

Un autre élément qui a son importance dans le choix d'une destination est le rapport des autorités locales face aux clandestins. Etre renvoyé dans son pays avant d'avoir amassé une épargne suffisante constitue un échec; il est donc nécessaire de n'être soumis qu'à une faible probabilité de se faire expulser. Pour les sans-papiers ${ }^{34}$, majoritaires parmi les migrants autonomes $^{35}$, plus que les législations, ce sont alors les modalités d'application des lois qui

\footnotetext{
${ }^{33}$ Sur le concept de « niche migratoire » voir notamment les travaux de R. Waldinger dont "The Making of an Immigrant Niche". Selon cet auteur, les réseaux migrants ont tendance à se déployer dans des sous-secteurs d'emplois qui leur ont été ouvert à un moment donné dans le pays dit d'accueil. La diffusion de l'information entre les migrants et les liens qui se tissent entre ces derniers et leurs employeurs tendent, par la suite, à maintenir et développer la niche d'emploi au sein du réseau initial.

${ }^{34}$ Même avant l'abolition du régime des visas, la majorité des voyages se faisaient légalement après l'obtention d'un visa de tourisme pour l'Espace Schengen. Toutefois, l'interdiction d'exercer une activité salariée et la restriction des séjours à une période de moins de trois mois font de ces migrants des clandestins dans les pays où ils travaillent.

${ }^{35}$ Par opposition à ceux qui sont recrutés sous contrat depuis la Roumanie.
} 
importent. Certains Etats, comme la France, l'Allemagne ou l'Autriche se montrent particulièrement rigides face aux migrants illégaux; tandis que d'autres, dont l'économie exploite massivement le travail au noir sont plus indulgents. L'étude menée dans la province d'Almeria est notamment un bon exemple de la gestion informelle à laquelle les migrations clandestines sont soumises : ici, les migrants ne sont pas arrêtés par la police durant les heures de repos agricoles; ils peuvent vaquer librement entre 12 et 16 heures. En revanche, les étrangers sont systématiquement arrêtés et contrôlés s'ils flânent dans des lieux publics durant les heures de travail. De cette façon, les autorités « trient» les migrants qui remplissent le rôle économique qui est attendu d'eux, de ceux qui chercheraient à quitter ce secteur. De même, les migrants clandestins savent que la complaisance des policiers s'arrête aux limites des régions agricoles. Voyager dans le reste du pays revient alors à quitter la zone d'indulgence qui caractérise la province d'Almeria. Ces observations montrent ainsi qu'au-delà des frontières nationales, il existe dans l'espace européen des enclaves qui seront plus propices à la constitution de niche migratoire.

\subsection{Une distribution réticulaire}

Les pratiques à l'œuvre dans ces migrations, faites de multiples savoirs et savoir-faire, et dont on a donné quelques illustrations dans les lignes précédentes, varient peu d'un réseau à l'autre; pourtant les publics qui y ont recours sont divers. Le cas des Tziganes est perçu comme spécifique dans la mesure où les raisons invoquées pour expliquer les départs sont autant culturelles, qu'économiques ou imputées aux discriminations subies en Roumanie. Même s'il existe un consensus populaire visant à catégoriser les migrations Tziganes comme la reprise du nomadisme interdit sous la dictature, n'ayant en cela rien de commun avec les pratiques migratoires de la population majoritaire dite "non-ethnique», on constate cependant que les deux phénomènes apparaissent en parallèles l'un de l'autre. Les pays le plus « visités » sont souvent les mêmes, à savoir l'Allemagne, la France, l'Italie, l'Espagne et plus récemment le Royaume-Uni. Les recours à la demande d'asile dans ces pays, que les Roms invoquent pour discriminations ethniques ${ }^{36}$ sont simultanément utilisés de façon manifestement infondée par les autres Roumains. Sans pousser plus en avant l'analyse, il apparaît cependant que, malgré certains points de contact, les réseaux d'entraide mobilisés par les uns et par les autres coïncident rarement. Il en est de même pour les autres groupes

\footnotetext{
${ }^{36}$ Voir à ce sujet l'étude de R.Benattig et O.Brachet sur les demandeurs d'asile Roms-roumains dans la région lyonnaise : Les dynamiques migratoires roumaines, le cas des demandeurs d'asile en France.
} 
minoritaires (Magyars, Allemands) qui, lors de leurs migrations, utilisent plutôt les ressources que leur offre leur appartenance ethnique.

Une autre distinction possible tient à l'origine rurale ou urbaine des populations migrantes. Depuis longtemps, les pays européens ont exploité, pour les travaux les plus ingrats des travailleurs étrangers peu qualifiés, souvent venus des campagnes de régions pauvres ${ }^{37}$. C'est sur cette catégorie de migrants qu'a porté l'enquête qualitative à laquelle j'ai participé dans la province roumaine de Teleorman ${ }^{38}$. En une dizaine de jour, trois équipes de recherche, soit douze personnes, ont tenté de dresser une monographie migratoire de trois villages de cette région. L'enquête a consisté d'une part en la diffusion d'un questionnaire à plus de cent personnes dans chaque localité puis a été complétée dans un deuxième temps par une soixantaine d'entretiens semi-directifs centrés sur des thèmes variés.

Depuis cette région, les migrations sont essentiellement orientées vers l'Espagne, autour de Madrid et dans la province d'Almeria. Les migrants concernés sont majoritairement des hommes, d'âges divers, dont le départ correspond à la volonté d'améliorer les conditions d'existence de leur famille restée au pays. Les personnes qui partent travailler en Europe de l'Ouest, ne sont pas issues des catégories les plus pauvres, qui ne possèdent pas les moyens de voyager. Il s'agit de ruraux de niveau social intermédiaire, appauvris par la transition qui souvent cumulaient, sous la dictature, une activité de paysan et un emploi dans une usine d'Etat. Leur premier départ implique des sacrifices pour les proches qui souvent aident à financer le voyage et l'achat du visa de tourisme au marché noir. Après quelques mois passés à l'étranger, les gains de la migration sont majoritairement investis en Roumanie dans la construction de demeures majestueuses, témoignant ainsi publiquement de la réussite de leur propriétaire. Lorsque, après quelques années durant lesquelles les séjours à l'étranger se sont succédés, on est parvenu à financer la construction de la maison, les bénéfices suivants, s'ils existent, serviront à l'achat de mobilier ou d'une voiture.

Les pratiques migratoires se diffusant de façon réticulaire, lorsqu'un village entre dans le processus cela touche une part importante de sa population. Ainsi, le travail à l'étranger se pratique intensément dans certaines localités à la différence d'autres communes qui ne font

\footnotetext{
${ }^{37}$ Voir notamment les travaux de M.Oriol sur l'immigration portugaise en France ou ceux d'A.Sayad sur l'émigration algérienne.

${ }^{38}$ L'enquête s'est déroulée du 6 au 14 juillet 2001. Elle était dirigée par le Professeur D.Sandu de l'Université de Bucarest. Le thème général en était: « Stratégies de vie dans le contexte communautaire : stratégies économiques et d'habitation ».
} 
aucun usage de cette solution. Cette manière de procéder rend la migration d'origine rurale très visible au niveau du pays puisqu'elle modifie la morphologie de villages entiers. Si bien que l'on a parfois l'impression que la migration est un phénomène qui se concentre essentiellement dans les campagnes ${ }^{39}$.

Pourtant, une autre catégorie sociale entre dans la partie et prend aujourd'hui le pas sur les autres. Il s'agit des jeunes citadins des classes moyennes. La migration de travail étant souvent illégale, on ne peut pas avoir de données quantitatives fiables à leur sujet. Néanmoins, les chiffres obtenus lors de sondages d'opinion soulignent certaines tendances. Ainsi, selon l'enquête du Baromètre d'Opinion Publique sus-mentionnée ${ }^{40}, 78 \%$ des personnes qui ont émigré puis sont revenues au cours des dix dernières années sont originaires de villes de plus de 30.000 habitants. Parallèlement, tandis que $18 \%$ de la population totale envisage d'aller travailler temporairement à l'étranger, parmi les 18-24 ans cette proportion est de $43 \%$ et de $34 \%$ chez les 25-34 ans. De même, on constate que les candidats au départ ont le plus souvent un emploi et une rémunération qui dépasse le salaire national moyen. Ces orientations générales, qui reposent sur des intentions et non des pratiques, justifient que l'on s'intéresse de plus près à ce public. C'est l'objet de l'enquête qualitative menée à Târgoviste, dont les résultats sont exposés dans les lignes qui suivent.

\section{La migration depuis Târgoviste}

Târgoviste est une ville de 100.000 habitants, située à 90 kilomètres de Bucarest. La population d'étude correspond bien, dans ses caractéristiques objectives, à celle identifiée par le Baromètre d'Opinion Publique. Tous les migrants rencontrés ont moins de 35 ans et la majorité a même moins de trente ans. Par définition, ils sont tous issus d'un milieu urbain Târgoviste est le chef lieu du département- et sont assez proches du centre névralgique du pays puisque leur ville n'est qu'à quarante-cinq minutes en voiture de la capitale. Par ailleurs, elle est relativement développée tant au niveau social qu'économique : elle abrite plusieurs usines et manufactures et se situe dans une région pétrolifère. La transition a, comme partout, conduit à des licenciements et réduit la production mais, comparé au reste du pays, la ville n'est pas dans une situation particulièrement précaire, son taux de chômage est aligné sur la

\footnotetext{
39 On constate à ce propos que les quelques recherches menées en Roumanie sur les migrations portent essentiellement sur des villages. Cf. Sociologie Romaneasca $n^{\circ} 2 / 2000$, consacré aux migrations roumaines ou les interventions de M.Serban et I.Muntele lors du séminaire « Les circulations migratoires roumaines après 1989 », Op. Cit.

${ }^{40}$ Fundatia pentru o societate deschisa, Op. Cit., p.46 et suiv.
} 
moyenne nationale. Récemment, une Université a été ouverte et plusieurs formations techniques supérieures existent depuis longtemps. On y trouve également un complexe sportif important et c'est un point de passage lors des tournées des artistes nationaux. De ce point de vue, il apparaît clairement que la migration n'est pas le fruit de la précarisation de la région. Elle émane d'un contexte plutôt favorisé.

Par ailleurs, la population concernée est elle aussi relativement bien pourvue face au milieu dans lequel elle évolue. La grande majorité des personnes rencontrées (plus de 80\%) avaient obtenu leur baccalauréat et la plupart ont entamé des études supérieures, soit dans une formation dispensée sur place soit à l'Université de Bucarest. Certaines d'entre elles ont terminé leurs études (c'est-à-dire ont achevé les quatre années minimales de formation), d'autres les ont abandonnées avant la fin du cursus. Dans la plupart des cas, elles ont ensuite exercé un emploi pendant quelques années. Dans le groupe étudié, les enseignants du secondaire étaient fortement représentés mais on y rencontre également des employés de bureau, des comptables, des juristes. D’autres sont issues de familles ayant déjà développé de petites entreprises privées auxquelles elles participaient. Des métiers manuels, comme garagistes ou ouvriers des usines environnantes apparaissent nettement moins souvent, bien que n'étant pas totalement exclus. Parmi l'ensemble des migrants interrogés, aucun ne s'est déclaré chômeur. En cela, ce qui distingue ces migrants de «l'émigration des cerveaux » tient moins à leurs qualifications, qui sont souvent d'un niveau relativement élevé, qu'à l'exploitation de leurs compétences au cours de la migration. Lors des enquêtes menées en France et en Grande-Bretagne, les sujets étudiés étaient toujours surqualifiés pour les postes qu'ils occupaient. Les emplois qui leur sont attribués, dans le bâtiment, l'hôtellerie ou l'agriculture, sont ceux traditionnellement réservés aux immigrés qui ne requièrent aucune compétence spécifique.

Les migrants sont issus de la très large classe moyenne roumaine. La plupart du temps, leurs parents avaient une profession intermédiaire ou supérieure, semblable à la leur, dans une entreprise d'Etat ou dans l'administration. Comme la majorité des jeunes roumains, avant leur premier départ, ces personnes vivent avec leurs parents, en «bloc ${ }^{41}$ dispersés dans les différents quartiers que compte la ville.

Socialement, leur position serait plutôt satisfaisante. Mais, étant donné la crise économique, ce statut ne correspond plus à des conditions matérielles convenables. On l'a dit,

\footnotetext{
${ }^{41}$ « bloc » est le terme roumain, particulièrement expressif, désignant les immeubles qui, dans les années 70 et 80, ont massivement remplacé les autres formes d'habitation en milieu urbain.
} 
ces dernières années, la classe moyenne qui, après quatre décennies de communisme, constitue l'essentiel de la population roumaine, voit ses conditions de vie se précariser au fil des ans, ce qui conduit ses jeunes à vivre en décalage avec leurs «habitus » de classe. Leurs aspirations, conséquence de leur socialisation dans un certain contexte social, ne sont plus en phase avec ce que la société peut aujourd'hui leur offrir. L'extrait d'entretien suivant illustre bien cet écart : «Moi j'ai du travail ici, je ne suis pas à plaindre, je n'ai pas envie de faire autre chose. Le problème c'est seulement mon salaire ; avec ce que je gagne, je ne peux pas vivre, je ne peux pas sortir quand je veux, aller au restaurant ou au cinéma; je ne peux pas m'acheter de nouveaux vêtements, je parle pas de partir en vacances... Moi je veux juste une vie normale, faire comme toi, comme tout le monde ici avant. C'est pour ça que je vais en France. C'est pas beaucoup, ce que je veux; c'est pas pour devenir riche ${ }^{42}$. A position sociale égale, ils n'ont pas la possibilité de suivre le même train de vie que leurs parents à leur age mais ils n'ont pas intériorisé ce déclassement. Cette dévaluation produit un sentiment de frustration indubitable. Malgré les réalités objectives, ils continuent à nourrir des ambitions similaires à celles de la génération précédente, et même entretiennent plus d'espoirs encore dans la mesure où la dictature ne restreint plus le domaine des possibles.

Un deuxième facteur renforce ce décalage entre pouvoir d'achat et aspirations : le passage au système capitaliste a engendré de nouveaux désirs. Après la Révolution, les Roumains ont imaginé qu'ils auraient en peu de temps accès à la société de consommation occidentale, depuis longtemps objet de convoitise. Le changement de système économique devait être accompagné d'une évolution positive des standards de vie. Comme l'explique notamment A.Portes, une des conséquences sociales de la mondialisation, illustrée ici par la chute du régime communiste, « est, dans les pays périphériques, le remodelage de la culture populaire d'après les modèles étrangers et l'introduction de modèles consuméristes sans rapport avec le niveau des salaires locaux». Il précise en outre que «paradoxalement, ce processus ne touche pas tant les classes les plus pauvres des sociétés périphériques que leurs classes moyenne et ouvrière qui sont généralement plus exposées aux messages publicitaires et aux symboles culturels projetés par les pays avancés ${ }^{43}$. Or, c'est bien de cette catégorie intermédiaire, la plus soumise à la culture de masse, que sont issus les migrants. Ce

\footnotetext{
${ }^{42}$ Entretien avec C., à Târgoviste, en avril 2000, traduit du roumain.

${ }^{43}$ Portes (A.), «La mondialisation par le bas », p.16. Ce thème est également abordé par différents auteurs concernant les migrations européennes, voir notamment M.Okolski, « La nouvelle donne migratoire en Europe de l'Est » ou C Withol de Wenden, L'Europe et toutes ses migrations.
} 
phénomène est amplifié ici dans la mesure où, de longue date, la Roumanie, île de latinité dans un monde slave, a orienté son regard, et ses espoirs, vers l'Occident. Aujourd'hui, les media et l'ensemble de la classe politique relayent cette tendance en prônant l'intégration des institutions européennes et euro-atlantiques ${ }^{44}$. L'occidentalisation est donc un processus ouvertement favorisé par une large majorité et, même s'il arrive aujourd'hui que l'on remette en question les bienfaits de la Révolution, peu de voix s'élèvent contre cette marche vers l'Ouest.

Dans ces conditions, avant même d'envisager de partir, les jeunes sont déjà sensibilisés aux attraits qu'est supposé offrir l'Occident. La migration vers ces pays apparaît comme une alternative au déclassement subit en Roumanie. D’une part, l'idée de travailler à l'étranger est perçue comme la possibilité de connaître une autre région du monde et d'avoir accès à la culture comme au mode de vie de l'Ouest. Les « émigrés », pour reprendre le terme employé par la société locale, ont le privilège de toucher du doigt, et même de participer à ces sociétés dites «civilisées ». Ce vocable est fréquemment employé pour désigner les pays industrialisés. Il s'oppose implicitement à l'Orient, dont la Roumanie cherche à se démarquer. Par ailleurs, l'expérience de ceux qui reviennent prouve que l'expatriation temporaire offre les moyens à ses acteurs de vivre, chez eux, fastueusement. Grâce aux gains accumulés mais aussi parce qu'ils importent un peu de la culture rencontrée à l'Ouest, ils font preuve d'une attitude plus dépensière qui apparaît comme le symptôme d'une nouvelle condition sociale.

L'objectif de la migration est rarement lié à la survie de la famille ou de la communauté d'appartenance, comme A.Sayad l'a décrit dans le premier âge de l'émigration algérienne $^{45}$. Souvent, les jeunes partent temporairement pour se donner les moyens de débuter une vie indépendante. Dans la plupart des cas, le premier départ est motivé par un projet précis qui doit trouver sa réalisation en Roumanie. Certains envisagent d'acheter un appartement ou une voiture, d'autres ont besoin d'un financement pour démarrer une affaire. Plus rarement, on part dans le but d'accumuler une réserve d'argent afin d'augmenter globalement son pouvoir d'achat. Les facteurs qui déterminent les personnes à entrer dans la migration sont donc essentiellement individualistes.

D’une certaine manière, la migration est également perçue comme véhicule autorisant le passage à l'âge adulte. Elle soutient en effet l'émancipation des jeunes qui trouvent là un

\footnotetext{
${ }^{44}$ Concernant l'attrait que représente l'Occident auprès de la population roumaine, voir Durandin (C.), « La course vers l'Ouest » in Roumanie, un piège, pp.105-138.

${ }^{45}$ Sayad (A.), « Les trois ages de l'émigration algérienne en France ».
} 
moyen de s'affranchir financièrement de la tutelle de leur famille. Ils acquièrent, en outre, au cours de l'expérience migratoire, un savoir sur le monde que les générations antérieures ne partagent pas et qui les distinguera lors de leur retour. En ce sens, pour les jeunes citadins, la migration possède une valeur tout autant symbolique que matérielle.

\subsection{Une sélection liée au capital social mobilisable}

Mais, bien que cette solution soit très prisée, elle reste difficile d'accès. Depuis Târgoviste, on migre essentiellement vers la France et la Grande-Bretagne. Le parcours modal, aujourd'hui, suppose que l'on se rende dans un premier temps à Nice puis, de là, on recherche un moyen de voyager jusqu'à Londres qui est actuellement la destination la plus appréciée. Bien que ce voyage ait été effectué par plusieurs centaines de personnes ${ }^{46}$, il reste réservé à un public averti. Le premier problème qui se pose était, jusqu'au début de l'année 2002, l'obtention d'un visa officiel pour l'Espace Schengen. La procédure normale de sollicitation était complexe et aléatoire. Elle nécessitait notamment de présenter une réservation d'hôtel ou un certificat d'hébergement ${ }^{47}$ dans le pays de destination et de fournir la preuve que l'on possédait les moyens financiers nécessaires à son autonomie durant toute la durée du séjour, documents difficiles à produire lorsque l'on est, en fait, candidat à l'émigration économique. Par ailleurs, l'autorité consulaire pouvait refuser la délivrance du visa sans obligation de motiver sa décision. Il s'agit donc d'une première épreuve sélective. Cette procédure était souvent court-circuitée grâce au marché noir. Les candidats au départ utilisaient, la plupart du temps, les services d'un intermédiaire. Celui-ci traitait directement avec les consulats afin d'acheter des visas régulièrement émis par ces derniers ou avec des faussaires. L'intérêt de ce relais tient à ce qu'il garantissait l'obtention rapide d'un droit d'entrée sur le territoire Schengen. Bien entendu, ce type d'entraide n'est pas gratuit, il est acheté au prix fort auprès de spécialistes. Ce qui implique, pour le migrant potentiel, d'investir une somme importante, entre 1000 et 1500 dollars, avant même d'avoir la certitude de rentabiliser son voyage.

Cependant, les ressources financières ne sont pas les seules à entrer en ligne de compte. Pour pouvoir acheter le droit de voyager, il faut d'abord savoir à qui s'adresser. Connaître les méandres du «marché de la migration » est un véritable enjeu. Sans relations

\footnotetext{
${ }^{46}$ D'après les migrants du même réseau interrogés au cours de l'enquête menée à Londres en octobre-novembre 2001, il y avait dans cette ville à ce moment-là plus de trois cents personnes originaires de Târgoviste (ces estimations variaient entre 200 et 500$)$.

${ }^{47}$ Document concédé par un habitant du pays de destination et validé par la municipalité de résidence de celui-ci.
} 
directes avec des personnes impliquées dans ces trafics, les solliciteurs ont toutes les chances d'être trompés, avec pour conséquences non seulement de ne pas pouvoir partir mais également d'être lésés de sommes importantes. Les histoires à ce sujet ne manquent pas parmi les migrants. L., qui vendait un journal de rue à Nice, raconte à ce propos : «Ca faisait plus d'un an que je voulais partir, mais j'ai eu des problèmes, chaque fois. Une fois, quelqu'un que connaissait mon cousin m'a dit qu'il pouvait avoir un visa pour moi. Il fallait que je paye -c'était pas trop cher- que je donne mon passeport et une semaine après, c'était fini, j'avais tout en règle. J'ai payé, la semaine d'après c'était pas prêt, j'ai attendu, attendu... Après deux mois, j'avais toujours pas de visa. J'ai dit à $X$ « Tant pis, maintenant je veux mon argent et mon passeport, tout de suite »Il a dit «d'accord». J'ai encore attendu. Au moins deux ou trois semaines après, il est venu avec mon passeport, mais l'argent, il me l'a pas rendu. Même maintenant, il me l'a toujours pas rendu. Je le verrai jamais cet argent. (...) Une autre fois, j'ai trouvé une firme, c'est quelqu'un que je connaissais qui m'avait dit -je travaillais avec lui-, là-bas, ils embauchaient des maçons pour l'Allemagne. J'ai payé deux mille dollars pour un contrat légal de six mois, avec le voyage, le logement, tout. Trois jours avant de partir, j'ai téléphoné ; personne m'a répondu. J'ai téléphoné pendant trois jours, parce que je savais pas où c'était le départ du car, mais 'y avait plus personne pour répondre, ils ont disparu, comme ça, je sais pas ce qui s'est passé avec eux ${ }^{48}$. Ils sont nombreux à avoir subi ce type d'échecs avant leur première migration réussie.

C'est pourquoi un élément essentiel dans la préparation de la migration clandestine est le capital social que les candidats peuvent mobiliser pour mener à bien leur projet. Les futurs migrants établissent un inventaire des personnes à même de les aider dans leur aventure. Comme le montrent K.Espinosa et D.Massey au sujet des migrations mexicaines aux EtatsUnis $^{49}$, les résultats atteints seront fonction de la qualité de ce soutien. Il apparaît en effet que lorsqu'un candidat au départ a déjà un membre de sa famille dans le pays où il souhaite se rendre, les échecs sont très rares. Au contraire, ceux qui, au moment où ils ont décidé de partir, n'ont aucun contact avec des migrants ou des «trafiquants » qui peuvent les soutenir, renouvellent plusieurs fois leurs tentatives et ont une période de latence plus longue, d'une année au moins. Ces délais et revers aboutissent parfois au renoncement. Entre ces deux extrêmes, les personnes qui choisissent de migrer ont souvent quelques amis plus ou moins

\footnotetext{
${ }^{48}$ Entretien avec L., au siège du journal Macadam, à Nice, en janvier 1998.

${ }^{49}$ Espinosa (K.), Massey (D.), "Undocumented migration and the quantity and quality of the social capital".
} 
proches auprès desquels elles vont solliciter de l'aide ou tenter d'obtenir des conseils. Selon la nature de ces relations, les services varieront en qualité et en coût.

Mais la nécessité d'appartenir à un réseau est loin d'être limitée aux obligations administratives de départ. Pour éviter de revenir bredouille après quelques mois, voire quelques semaines dans les cas les plus équivoques, il est utile de ne pas partir à l'inconnu. Le soutien apporté par autrui peut intervenir sous deux formes : l'une concerne la communication d'informations utiles; l'autre est le fruit de l'aide directe que peut apporter le groupe. Rares sont les personnes qui quittent leur pays sans avoir un minimum de connaissances sur leur destination et les possibilités de travail et de logement qu'elle offre. Or, pour apprendre, par exemple, les secteurs dans lesquels il est possible de se faire employer à Londres ou bien où l'on peut louer une chambre à Nice quand on est irrégulier, il est indispensable de côtoyer directement des personnes qui se sont déjà rendues sur place ou qui ont d'étroites relations avec celles-ci. C'est pourquoi le premier souci d'un migrant potentiel sera de se connecter au réseau existant de migrants. Etant donné que les migrations sont faites d'incessants allers et retours, il y a toujours, sur place, à Târgoviste, un groupe plus ou moins important de personnes qui détiennent des indications utiles. Pourtant, les migrants n'en feront part qu'à leurs proches ou à des interlocuteurs dignes de confiance. De même, lors des séjours à l'étranger, il n'existe pas de soutien inconditionnel de la communauté roumaine. L'aide apportée aux nouveaux venus s'octroie toujours sur la base de relations personnelles et de recommandations. Or, celle-ci s'avère très utile dans le contexte hostile de la migration clandestine. Car même avec l'autorisation récente de voyager, les Roumains ne peuvent rester légalement plus de trois mois en Union Européenne et ne bénéficient pas d'un droit de travail. C'est donc par l'intermédiaire des milieux informels qu'ils pourront s'insérer dans l'économie des lieux qu'ils investissent. La réussite de la migration, c'est-à-dire le fait d'atteindre son objectif de départ, dépend alors fortement de la position du migrant dans cet environnement.

Sans le formaliser, chacun est conscient de la nécessité de posséder des relations. C'est la qualité de ce capital social, autant que son étendue, qui conditionnent le succès de la migration, et la décision même de se lancer dans l'aventure. Cette façon de faire explique que certains groupes soient particulièrement mobiles. On trouvait notamment une proportion importante de professeurs de sports parmi les Roumains rencontrés à Nice. Après enquête, cela n'est pas à attribuer à des données objectives particulières qui pousseraient ces enseignants à migrer. L'explication tient au fait que les premiers à avoir «exploré » la destination niçoise relevaient de cette formation. A leur retour, ils ont communiqué leurs savoirs sur ce lieu aux amis avec lesquels ils avaient le plus de contacts, c'est à dire à leurs 
collègues, anciens camarades d'université, qu'ils côtoyaient régulièrement à la salle de sport. Ce n'est que plus tard que cette étape migratoire s'est peu à peu ouverte à un public plus large. Mais, encore aujourd'hui, les personnages centraux de ce réseau, aujourd'hui tourné vers Londres, sont issus du milieu sportif.

On remarque, par ailleurs, que la migration elle-même permet de multiplier ses relations. En effet, participer, à l'étranger, à un réseau de solidarité devient rapidement une carte de visite. L'intégration dans le cercle des migrants qui, au départ, n'est pas favorisée par les membres du groupe, se transforme bientôt en un atout que l'on peut faire valoir auprès d'autres participants au réseau. Contrairement au «protectionnisme» dont ils font preuve dans leur ville d'origine, en situation migratoire les migrants collaborent avec moins de retenue. Même en quittant la Roumanie avec des contacts à l'étranger, un nouveau migrant est toujours relativement isolé à ses débuts. Puis, faisant sa place dans le milieu, le développement de ses relations personnelles lui ouvre de plus en plus de portes. Parallèlement, les différentes expériences lui procurent un savoir et un savoir-faire grandissant. La migration devient ainsi, dans le temps, une situation de plus en plus confortable. De ce fait, tandis que le premier départ était peu assuré et source de nombreuses hésitations, les suivants demanderont un investissement bien moindre.

\subsection{L'installation dans la mobilité}

Cela explique en partie l'installation dans la mobilité. Tandis que le premier voyage, limité dans le temps, soutenait un projet particulier, la migration, faite de continuels allersretours, devient par la suite une fin en soi. Ce qui était perçu comme une épreuve momentanée apparaît désormais comme une alternative à la vie sédentaire. Son attrait est d'autant plus fort que les migrants adoptent, grâce à leur séjour à l'étranger, un nouveau mode de vie qu'il leur sera difficile d'abandonner. Après avoir durement travaillé à l'Ouest, ils profitent pleinement, chez eux, de leur nouvelle situation. Leurs dispositions financières changent radicalement leurs habitudes ; ils ont alors la possibilité de pratiquer des loisirs, de voyager dans le pays, de s'adonner à l'oisiveté et ne s'en privent pas. Si bien que les gains qui devaient servir à acheter un appartement ou monter une petite société sont finalement mis au service d'une vie facile.

Même si le projet est altéré, la migration procure une certaine aisance qui devient alors l'objet même de la circulation continue. Car, après avoir découvert un mode de vie agréable et nourri de nouvelles ambitions, ces « nouveaux riches » ne sont pas enclins à renouer avec leur ancienne condition. Un jeune couple explique : «La première fois, il est parti seul, c'était pour acheter notre appartement, parce qu'on habitait avec mes parents. On l'a acheté, puis on s'est dit que s'il restait encore un peu, on pourrait acheter une voiture, pour là-bas. Après, 
on a pensé que ce serait bien de louer l'appartement et d'acheter une maison pour nous, parce que c'est mieux, une maison, on peut avoir un jardin. Pour ça, il est parti la deuxième fois à Londres, parce qu'ici [à Londres] on savait qu'on gagnait plus. Et puis, on a pensé à faire une maison plus grande, pas une petite maison, une grande. Alors je suis venue moi aussi, pour travailler aussi, et pour être avec lui, parce que ça faisait déjà deux ans qu'on n'était plus ensemble. Mais maintenant je suis vraiment saturée de l'étranger. Je veux rentrer chez moi. Je ne veux plus avoir de nouvelles idées, on prend ce qu'on a et on fait avec, c'est tout. $\gg{ }^{50}$ Au-delà, la situation même de migrant contribue à faire évoluer les grilles de lecture de ces jeunes. Comme le souligne M.Okolski, la migration a pour conséquence «l'adoption de mentalités, d'attitudes, de comportements et d'habitudes modernes par les immigrants provenant de pays moins « modernes » " ${ }^{51}$, le processus de « modernité » devant être compris ici comme une acculturation à la société de consommation. Leurs comportements, calqués sur de nouvelles normes, sont endossés pour une part volontairement mais certains sont intériorisés de façon inconsciente. Le modèle observé à l'étranger et la nouvelle position sociale qu'ils occupent à leurs retours constituent désormais des références. Voici la réponse de l'un d'eux lors d'un entretien : «Depuis ton retour, as-tu repris ton ancien emploi ou une nouvelle activité salariée?

-Travailler! Moi! Ici! C'est une blague? Tu as une idée des salaires ici? Si je travaille pendant un mois, huit heures par jour, avec un chef qui m'engueule toute la journée, je gagnerai autant qu'en une après-midi à faire les pare-brise à la sortie de la voie rapide [à Nice]... Non, je ne travaille pas ici, je me débrouille, je fais des affaires ${ }^{52}$. On comprend bien, à travers ce type de discours, l'ubiquité dont est sujet le migrant. Il compare son salaire ici et là-bas comme s'il pouvait, à tout moment, choisir entre l'un et l'autre. La distance et les difficultés liées à la migration sont totalement absentes du discours. Sa lecture repose sur une position intermédiaire entre deux aires géographiques : l'avantage de «faire les pare-brise » à l'Ouest tient non pas aux gains importants engendrés par cette activité en France mais au fait qu'il compare ces gages aux salaires perçus en Roumanie. C'est cette appartenance multipolaire qui conduit à ne plus accepter le seul éventail des possibilités qu'offre son pays d'origine.

\footnotetext{
${ }^{50}$ Entretien avec O. et B., en novembre 2001 à Londres, traduit du roumain.

${ }^{51}$ Okolski (M.), Op. Cit., p.30.

${ }^{52}$ Entretien avec I., à Pucioasa, le 26-05-2000, traduit du roumain.
} 
Le migrant devient alors acteur d'une mobilité permanente. Tandis que la première fois, il avait un programme à réaliser, il adopte par la suite une façon de vivre qui le lie en même temps à sa ville d'origine et à l'étranger qui, à ses yeux, constitue une seule et même entité. L'ailleurs qui lui est ouvert est constitué d'une somme de localités dans lesquelles il sait pouvoir trouver une assistance. Ces points sont eux-mêmes liés par des savoirs multiples : savoir où se faire employer, savoir comment voyager d'un lieu à l'autre, savoir négocier avec les autorités locales, etc. Pour reprendre les termes d'A.Tarrius décrivant les pratiques nomades des commerçants d'origine étrangère à Belsunce, les membres d'un même réseau migrant dessinent, par leurs multiples pratiques, un vaste territoire circulatoire $^{53}$. Le migrant a, à sa disposition, une cartographie des lieux domestiqués par le réseau. En contribuant à son évolution, il obtient un laissez-passer dans cet espace symbolique qui lui permet d'entreprendre, avec de moins en moins de contraintes, des séjours temporaires à l'étranger.

Toutefois, le potentiel migrant n'est pas uniforme ; il varie selon les circonstances. Plus une personne est expérimentée, plus son territoire est étendu. La particularité de chaque lieu détermine également les pratiques migratoires qui y sont associées. On a vu que la rigueur des contrôles effectués sur les personnes ou auprès des employeurs était un point important dans la constitution de niche migratoire. D'autre part, la proximité géographique, autorise de fréquents déplacements ; c'est le cas de l'Italie qui, étant aux abords de l'Espace Schengen, n'est pas difficile à atteindre (par la Serbie-Monténégro notamment pour laquelle les Roumains n'ont pas besoin de visas). La faible distance qui sépare les deux pays réduit aussi le coût du voyage, même s'il doit être clandestins. De ce fait, cette destination offre la possibilité de rentrer régulièrement en Roumanie tout en envisageant de revenir par la suite. Car la durée des séjours à l'étranger dépend notamment de la possibilité de repartir en cas de retour chez soi. La Grande-Bretagne est, en ce sens, une destination qui immobilise les migrants. N'appartenant pas à l'Espace Schengen, sa configuration insulaire est un rempart efficace contre les migrations clandestines. Les Roumains savent que s'ils quittent Londres, il leur sera difficile de s'y rendre à nouveau. C'est pourquoi ils réduisent leurs circulations après avoir atteint ce lieu. Certains, bien qu'ayant le projet de rentrer en Roumanie, où ils

\footnotetext{
${ }^{53}$ Par le concept de territoire circulatoire, cet auteur entend, dans une approche paradigmatique de la mobilité, «investir le mouvement spatial de sens social». Au delà de l'idée de flux entre des sédentarités, «la notion de territoire circulatoire constate la socialisation d'espaces supports à des pratiques de mobilité », et considère que «les individus se reconnaissent à l'intérieur des espaces qu'ils investissent ou traversent au cours d'une histoire commune de la mobilité, initiatrice d'un lien social original». Extraits de Tarrius (A.), «Le lien social fort comme préalable à la réussite économique ».
} 
investissent leurs économies, sont sur le sol britannique depuis plus de deux ans, tandis que la durée moyenne de séjour à l'étranger est d'environ une année.

\subsection{Les « émigrés », un groupe social en construction}

De même, le temps passé en Roumanie lors des retours est flexible. Les périodes concernées s'étendent de quelques mois à plusieurs années. Cela dépend en partie de l'investissement qui est fait dans le pays. Il arrive que des migrants lancent une affaire. Il peut s'agir de sociétés de transport marchand, secteur en pleine expansion, ou de petits commerces. Le patron est alors tenu de rester sur place, pour veiller à la bonne marche de l'entreprise. Mais, est-ce dû à la conjoncture économique ou au manque d'expérience de leurs créateurs, ces petites entreprises ne résistent jamais longtemps ; la majorité ferme ses portes après une année au plus. D'autres s'exercent à de petits trafics ; leur capacité financière lors des retours les autorise pendant un certain temps à faire fructifier leur argent en investissant sur des affaires ponctuelles, rachetant par exemple un lot de machines d'une usine en faillite pour le revendre auprès d'artisans. Ces activités leur permet de faire perdurer un moment leur capital, sans l'empêcher pourtant de s'épuiser. D'autres encore, ceux qui sont déjà partis plusieurs fois, ne rentrent que pour prendre du repos, leurs séjours sur place se réduisent alors à quelques mois.

Mais quel que soit la durée de leurs séjours chez eux, les migrants ont une place importante dans leur ville d'origine. Certains ont une vie de famille à Târgoviste. Dans ces cas, il n'est pas rare que la femme, après un certain temps, rejoigne son mari à l'étranger pour des périodes plus ou moins longues, les enfants étant alors confiés aux grands-parents. La situation inverse, dans laquelle la femme part avant, voire sans, son mari est moins fréquente mais il arrive parfois qu'une mère de famille migre momentanément dans un pays du sud, comme l'Espagne ou l'Italie, où l'emploi clandestin féminin, particulièrement le travail domestique, est plus développé. Même si son mari reste en Roumanie avec d'éventuels enfants, le départ d'une femme est généralement corrélé à celui d'un homme proche, frère ou cousin. Ce n'est qu'après une première migration de ce type que quelques-unes d'entre elles évoluent seules dans le territoire circulatoire du réseau ${ }^{54}$. Mais dans tous les cas, les femmes restent étroitement liées à leur famille. Cela s'illustre notamment par la fréquence importante des appels téléphoniques vers leur famille (jusqu'à deux fois par semaine), tandis que les hommes non mariés téléphonent plus rarement. Parmi les hommes, ceux qui partent

\footnotetext{
${ }^{54}$ Il n'est pas fait mention ici des migrations liées à la prostitution qui seraient peut-être justement le moyen de s'enrichir à l'étranger pour des femmes ne possédant aucun capital social migrant.
} 
célibataires rentrent parfois pour se marier. C'est ce que prévoit N. qui, entre différents voyages, a passé trois années à Nice puis deux en Grande-Bretagne : «Maintenant, il faut que je rentre en Roumanie. J'ai 31 ans, les types qui habitent avec moi ici [à Londres], ils sont tous plus jeunes que moi, et ils sont tous mariés ou presque. Pas lui, mais il a que 20 ans. Moi, maintenant il faut que je fasse une famille. Je vais rentrer en Roumanie, je vais rencontrer une fille bien, je me marie, et après... on verra. Peut-être j'irai en Italie, ou en France, j'aime beaucoup la France. J'ai des copains à Marseille. Mais je sais pas, d'abord il faut aller en Roumanie, trouver une fille qui m'aime... ${ }^{55}$. On constate à travers ce type de discours que, malgré le temps passé à l'étranger, seule la ville d'origine reste l'objet d'un ancrage social à long terme.

Cette affirmation sur place s'illustre aussi dans les acquisitions faites par les migrants. Ils achètent des appartements, qui deviennent leur résidence principale, des voitures qu'ils ramènent de l'étranger, etc. De sorte que tout ce qu'ils possèdent se trouve ici, leur mobilité à l'étranger ne leur permettant pas d'y accumuler quoi que ce fût. Ainsi, le temps passé ailleurs ne contrarie nullement l'installation sur place, au contraire, la migration permet d'affirmer plus ostensiblement sa présence dans la ville.

Car le groupe des migrants n'est pas seulement une construction théorique en vue de circonscrire une somme d'expériences particulières. Le vocable d' "émigré », dans le langage courant, désigne une catégorie clairement identifiée. Pour comprendre cette définition, il faut s'intéresser tant à la perception de ce groupe par la société sédentaire qu'à la façon dont les migrants eux-mêmes se mettent en scène.

Cette dernière passe notamment par l'espace public dans lequel ces jeunes gens exposent leur identité d'émigré. Leur apparence physique est le premier élément de distinction. De leurs voyages, ils ramènent différentes panoplies typiquement occidentales, en phase avec les dernières modes de ces pays ou simplement griffées de marques inabordables en Roumanie. Un T-shirt près du corps, accompagné d'un «vrai Levi's » (à distinguer des contrefaçons turques) et d'une paire de baskets Nike, sont ainsi les marqueurs indubitables d'un passé à l'étranger. De même, les voitures achetées à l'Ouest portent toujours le macaron du pays d'où elles ont été importées et, dans la mesure du possible, on gardera également l'immatriculation étrangère. Dans le même registre, en public un migrant n'hésitera pas à employer quelques mots ou quelques jurons en français, montrant par-là qu'il a intériorisé le

\footnotetext{
${ }^{55}$ Discussion avec N., à Slough, banlieue londonienne, novembre 2001.
} 
langage du pays duquel il revient. L'ensemble de ces signes extérieurs de migration propose, à l'initiative de ses acteurs, un certain stéréotype du migrant.

Parallèlement, les loisirs de ces derniers sont typiques de la condition des « émigrés de retour au pays ", d'où qu'ils soient. D’une façon générale, ils sont plus dépensiers que leurs compatriotes. Ainsi, aux yeux de la population locale, la vie facile, la fréquentation quotidienne des lieux de détente comme les cafés, restaurants ou discothèques est un trait caractéristique de ce public. Plus précisément, à Târgoviste, certains de ces établissements sont l'apanage des migrants. Leur décoration, la musique qui y est diffusée, les terrasses en extérieur rappellent les bars ou les discothèques occidentales. Les tarifs proposés sélectionnent la clientèle, écartant ainsi une majorité de la population locale. Garant leurs voitures rutilantes devant, les migrants s'y retrouvent en groupe plusieurs fois par semaine. Se rendre régulièrement à ces rendez-vous conduit dès lors à se faire reconnaître comme migrant tant par ses «collègues» que par les non-migrants. Car si toutes les personnes qui ont travaillé à l'étranger n'ont pas vécu leurs déplacements ensemble, leurs pratiques communes tendent ici à les rapprocher. Par exemple, si l'un d'eux prend des vacances aux sports d'hiver, il est probable qu'il s'y rende avec d'autres migrants, ses amis sédentaires n'ayant certainement ni les moyens ni le temps de l'accompagner. Cette constitution du groupe s'opère de la même façon dans les affaires. Les conditions requises par ce milieu, disponibilité en temps comme en liquidités, conduisent à s'associer en premier lieu avec des pairs. De cette façon, le groupe a tendance à se replier sur lui-même et, par là-même, à rendre public ce découpage in/out.

De l'extérieur, ces comportements peuvent apparaître ségrégationnistes. R. explique ainsi, tandis que je lui demandais s'il avait des contacts avec des migrants: «J'ai beaucoup d'amis qui sont partis partout, en France, en Italie, en Espagne... Mais quand ils reviennent, ce ne sont plus des amis. Ils ne te parlent plus, ils deviennent hautains. Au début, je leur téléphonais, je faisais comme avant. Mais eux, ils sont riches maintenant, ils ne sont plus intéressés par des gens comme moi. Ils me regardent de haut. C'est comme ça, quand les gens partent, ils ne sont plus pareils quand ils reviennent ${ }^{56}$. Il est certain que ce type d'attitude, assez répandu, contribue à forger une image dépréciative -bien qu'enviée- des migrants dans la société locale. Parallèlement, une façon de dénigrer ces derniers est de s'interroger sur «ce que cache en fait la migration $»^{57}$, insinuant que ces jeunes parvenus sont peut-être impliqués

\footnotetext{
${ }^{56}$ Discussion avec R. à Târgoviste, mai 2000, traduit du roumain.

${ }^{57}$ Commentaires émis par une étudiante au sujet de mes recherches sur les migrations, avril 1999, Târgoviste.
} 
dans des trafics inavouables que la fréquentation de l'étranger ne servirait qu'à masquer. Cependant, le stigmate négatif n'est qu'une partie de l'exo définition du groupe. La population comprend également, en observant leurs comportements, que la migration est un tremplin à l'ascension sociale, si difficile en période de crise. On retient qu'après six mois à l'étranger, les personnes de retour évoluent dans des sphères sociales prestigieuses. En cela, bien qu'elle soit souvent dénoncée avec amertume, l'attitude "hautaine » des migrants participe à la construction du mythe de la migration.

Cette illusion n'est d'ailleurs jamais démentie par les membres du groupe. Les difficultés, l'abnégation de soi dont il faut parfois faire preuve lorsque l'on «travaille» à l'étranger ne sont jamais abordées. A l'époque où Nice était une destination privilégiée, l'occupation principale des migrants consistait en la vente des journaux de rue, réservée en France à une population économiquement et socialement marginalisée. Pour un enseignant, un comptable ou un artisan, ce type de déclassement, même temporaire, est particulièrement dévalorisant. Mes interlocuteurs soulignaient ce point avec insistance lors des entretiens réalisés en France. En revanche, le sujet est évité dans la ville d'origine. Concernant la migration niçoise, on raconte par exemple lors des retours que l'on était vendeur de journaux, mais l'on ne précise jamais les conditions sociales de cette activité. Au cours d'entretiens menés à Nice, plusieurs nouveaux migrants ont fait part de leur déception en découvrant la réalité quotidienne. Les commentaires de D. sont explicitent sur ce point. Ayant obtenu un statut légal en France, elle a procuré un certificat d'hébergement pour son amie qui insistait pour travailler à l'étranger : «C. est très déçue de la France. Ca fait longtemps que je lui disait qu'ici c'est pas comme elle rêve, pas du tout! Le problème c'est que, moi par exemple, quand je rentre en Roumanie, je suis comme une reine. Elle voit que je rentre dans un bar, que je paye pour tout le monde, je suis la personne la plus importante. Et on fait tous comme ça quand on rentre. Alors, elle, elle croyait qu'on vivait comme ça en France, elle croit qu'ici on a une vie de roi. Mais elle savait pas qu'ici on travaille comme des chiens! Elle sait pas que pour faire ça pendant une semaine en Roumanie, il faut qu'on économise toute l'année ici. Elle sait pas qu'ici on sort même pas une fois par mois. Elle croyait qu'ici aussi on était des rois, qu'ici tout le monde vit comme ça.(...) Mais moi je peux te dire que quand on est ici, on n'est pas fier de ce qu'on fait. $"{ }^{58}$ Ces propos soulignent bien le rapport conscient qu'il existe entre l'admiration portée aux migrants et l'occultation des conditions de vie qui sont les leur à l'étranger. C'est pourquoi le tabou sur cette question est collectivement respecté par les

\footnotetext{
${ }^{58}$ Entretien avec D., 12 décembre 2000, à Nice.
} 
migrants en Roumanie. Ils savent qu'en valorisant l'attrait de la migration auprès de la population locale, ils contribuent à parfaire leur propre représentation.

Ce jeu de construction d'image et de redéfinition de la part des acteurs de la migration comme des «sédentaires» dessine les contours d'un nouveau groupe social reconnu par l'ensemble de la population. Si elles ne s'accordent pas unanimement sur sa définition, les différentes parties, par leur activité de catégorisation, témoignent de l'existence d'une entité à laquelle on attribue certaines caractéristiques. En ce sens, être migrant ne correspond pas simplement à une activité ; cela revient, au même titre qu'une profession, à acquérir un statut particulier dans la société locale. En ce sens, les migrants constituent une catégorie de « nouveaux riches » qui, bien que se dissociant des classes moyennes, ne doit pas sa réussite à une activité socialement blâmable, se différenciant par là-même de la mafia ou des milieux politiques, autres canaux classiques d'enrichissement. Par ce biais, ces jeunes gens complètent en quelque sorte le rêve d'ascension sociale offert par le capitalisme et ce, malgré la conjoncture économique nationale.

\section{Bibliographie}

Aktar C. et Ögelman N., "Recent Developments in East-West Migration: Turkey and the Petty Traders", in International Migration, Vol.32, 1994, Genève, IOM, pp.343-353.

Asociata Româna de Sociologie, Sociologie Romaneasca -Migratie, serie noua $n^{\circ} 2 / 2000$, Bucarest.

Bartram (D.), "Foreign workers in Israel: History and Theory" in International Migration Review, New-York, vol.32, $\mathrm{n}^{\circ} 2$ summer 1998, pp.303-325

Benattig (R.), Brachet (O.), Les dynamiques migratoires roumaines, le cas des demandeurs d'asile en France, Lyon, DPM, 1998.

Bourdieu (P.), «Le capital social» in Les actes de la recherche en sciences sociales, Paris, $\mathrm{n}^{\circ} 31$, janvier 1980, pp.2-3.

Colonomos (A.) (ss dir.), Sociologie des réseaux transnationaux, Paris, L'Harmattan, 1995.

Diminescu (D.), Lagrave (R.), «Faire une saison. Pour une anthropologie des migrations roumaines » in Migrations Etudes, n91, Novembre-décembre 1999.

Duchêne (G.), "Les revenus informels en Roumanie: estimation par enquête " in Revue d'études comparatives Est-Ouest, vol.30, n4, 1999, pp.35-64.

Durandin (C.), Roumanie, un piège?, Editions Hesse, Collection Ister, 2000. 
Espinosa (K.), Massey (D.), "Undocumented migration and the quantity and quality of the social capital" in Sozial Welt. Sonderband, Baden-Baden, Nomos, vol.12, 1997, pp.141162.

Fundatia pentru o societate deschisa, Barometrul de opinie publica, Cluj-Napoca, Metro Media Transilvania, Mai 2001.

Kideckel (D.), The Solitude of Collectivism, Cornel University Press, 1993.

Lhomel (E.), « Roumanie 2000-2001. Un nouveau départ?» in Le courrier des pays de l'Est, $\mathrm{n}^{\circ}$ 1016, juin-juillet 2001, Paris, La documentation française, pp.164-177

Ma Mung et Al., La circulation migratoire. Bilan des travaux-Migrations études, n84, 1998.

Michalon (B.), « De la politique des Aussiedler à la circulation : diversification des pratiques migratoires des Saxons de Transylvanie », à paraître.

Morokvasic (M.), «La mobilité transnationale comme ressource: le cas des migrants de l'Europe de l'Est» in Cultures et Conflits, n³3-34, Printemps-été 1999, pp.75-125, (http://www.conflits.org).

Morokvasic (M.), Rudolph (H.) (ss dir.), Migrants. Les nouvelles mobilités en Europe, Paris, L'Harmattan, 1995.

Okolski (M.), «La nouvelle donne migratoire en Europe de 1'Est » in Revue européenne des migrations internationales, vol.8, $\mathrm{n}^{\circ}$ hors série, 1992, pp.7-38.

Piore (M.J.), "The Shifting Grounds for Immigration" in The Annals of the American Academy, Mai 1986, pp.23-33.

Portes (A.), « La mondialisation par le bas » in Les actes de la recherche en sciences sociales, $\mathrm{n}^{\circ} 129$, Seuil, septembre 1999, pp.15-25.

Portes (A.), "Social capital: its origins and applications in modern sociology" in Annual Review of Sociology, vol.24, 1998, pp.1-24.

Sandu (D.), «Les enjeux des réseaux migratoires dans l'espace social de la transition », Communication orale, Maison de Sciences de l'Homme, Paris, 4 avril 2001.

Sayad (A.), "Les trois ages de l'émigration algérienne en France » in Les actes de la recherche en sciences sociales, juin 1977, pp.59-79.

Selim (M.), «Entretien avec A.Liiceanu: De la décommunisation au capitalisme en Roumanie » in Journal des Anthropologues, vol.77-78, AFA, 1999, pp.53-65.

Tarrius (A.), « Le lien social fort comme préalable à la réussite économique » in Journal des Anthropologues, vol.84, AFA, 2001, pp.111-144. 
Tarrius (A.), «Territoires circulatoires et espaces urbains» in Annales de la recherche urbaine, $\mathrm{n}^{\circ} 59-60$, juin-septembre 1993, pp.50-60.

Verdery (K.), What was socialism and what comes next, Princeton, Princeton University Press, 1996.

Waldinger (R.), "The making of an immigrant niche" in International Migration Review, New-York, vol.8, n¹ spring 1994, n¹1994, pp.3-30.

Wihtol de Wenden (C.), De Tinguy (A.), L'Europe et toutes ses migrations, Bruxelles, Editions complexes, 1995. 